\title{
Téoros
}

Revue de recherche en tourisme

\section{L'hiver québécois : un produit touristique}

\section{Louise Alex Trottier}

Volume 8, numéro 3, novembre 1989

Tourisme hivernal

URI : https://id.erudit.org/iderudit/1080309ar

DOI : https://doi.org/10.7202/1080309ar

Aller au sommaire du numéro

Éditeur(s)

Université du Québec à Montréal

ISSN

0712-8657 (imprimé)

1923-2705 (numérique)

Découvrir la revue

Citer cet article

Trottier, L. A. (1989). L'hiver québécois : un produit touristique. Téoros, 8(3),

19-25. https://doi.org/10.7202/1080309ar d'utilisation que vous pouvez consulter en ligne.

https://apropos.erudit.org/fr/usagers/politique-dutilisation/ 


\section{L'hiver québécois: Un produit touristique}

"Mon pays ce n'est pas un pays, c'est l'hiver..." Gilles Vignault
L'hiver résonne bien au coeur des Québécois: neige, froidure, poudrerie, sports, plaisirs, il n'a aucun secret pour nous; mais j'ai pu constater qu'il en va parfois autrement pour un "étranger". On peut alors se demander si cette saison a suffisamment d'attraits pour se qualifier en tant que "produit touristique"?

En 1982, lors d'un voyage d'agrément, comme on dit dans les statistiques, je me retrouvai aux Saintes-Marie-de-la-Mer, dans une boutique, en train de parler de mon pays, plus particulièrement de l'hiver, à quelques Méditerranéens.

Ainsi, je leur dis qu'après une tempête de neige, si on veut sortir, on doit pelleter le devant de sa porte de maison; qu'on doit souvent porter mitaines et foulard, pour éviter de se geler les doigts et les oreilles. En voyant leurs yeux s'écarquiller en entendant ces faits, si banals pour moi, je radoucis le discours, en disant que jamais on ne souffrait du froid dans nos maisons, très bien chauffées et que nos autos étaient bien adaptées au climat.

Quand je repense à cet épisode, je me souviens d'avoir affirmer mon identité culturelle de nordique québécoise avec beaucoup de vigueur, mais je doute du goût que $\mathrm{j}^{\prime}$ ai pu leur donner de venir palper cette saison, sur le terrain même du Québec, $\mathrm{j}$ "ai plutôt créé certaines appréthensions. Mais au fait, est-ce vraiment mon discours qui fut inquiétant ou n'est-ce pas plutồt la réalité hivernale tout simplement?

Pour répondre àcettequestion, il m' apparâtt important de faire un détour parl'ethnologie, qui nous montrera comment l'hiver a tracé ses sillons dans notre sociêté. J'examinerai brièvement d'abord le rapport passế et présent des Québécois à leur hiver, ainsi que celui qui se dessine pour le futur. Je discernerai donc une première étape, qui fut celle des ruptures et de la survivance; une seconde, qui se rapporte à l'aménagement et aux plaisirs de l'hiver; enfin, une troisième qui examinera l'impact de l'effet de serre sur notre culture hivernale. Je terminerai ce court essai en situant le Québec hivernal dans le cadre du système des attractions touristiques, suivant l'approche de Dean Mac Cannelll.

\section{De la survivance à la domestication}

Lorsque nos ancêtres sont venus s'installer sur les bords du Saint-Laurent, ils méconnaissaient complètement l'hiver et ce, malgré les avertissements que les Amérindiens leur avaient donnés. En effet, ces Français ne pouvaient se représenter l'hiver dans toute sa rigueur, au mois de juillet; pas plus que deviner que l'automne est une entre-saison où on doit hâter les préparatifs de l'hiver. Longtemps, il y eut en Nouvelle France l'idée qu'il n'y avait que deux saisons: "on n'en compte proprement que deux, car nous passons tout d'un coup d'un grand froid à un grand chaud, et d'un grand chaud à un grand froid; $c^{+}$est pourquoi on ne parle que par hiver et par étét".

La lutte contre l'hiver a dû être un combat extrêmement vif, sur tous les plans; elle comportait d'abord la rupture d'avec l'ancien mode de vie européen, et ensuite la création d'un nouveau, basésurl'adaptation au climat qui était une question de survie, l'hiver étant synonyme de maladies, d'épidémies et de mort mais également d'isolement; les gens "s $s$ "encabanaient" littéralement.

\section{Les ruptures}

Les Français, arrivant en Amérique, ont dû changer rapidement leurs habitudes de vie: en architecture, d'une part, leurs maisons de pierres européennes ne convenaient pas du tout au climat, elles gardaient le froid et l'humidité, d'autre part, ils furent incapables de prendre les modèles autochtones, maisons d'écorce, cabanes, trous dans la neige. Ils construisirent donc des maisons de bois; elles protégeaient davantage du froid et le matériau y était en abondance. Ces maisons furent moins espacées et situées en bordure des chemins afin de faciliter le déneigement. Les premiers habitants ont dô emprunter la traîne et la raquette, puis-
*Madame Louise Alex Trottier est consultante en tourisme et chargèe de cours a l'UOAM. 
que ce n'est qu'au 18 e siècle qu'on fit venir des chiens et les chevaux qui assureront les déplacements surun réseau de routes saisonnières, rattachées au chemin du roi.

Tout le domaine de l'habillement fut également touché: "Malgré la froidure hivemale, les pionniers de la Nouvelle France s"attachent à leur mode vestimentaire européenne... ce n'est que par nécessité absolue qu"ils adoptent le costume autochtone" ${ }^{n 3}$.

\section{La domestication}

"La domestication de 1'hiver marque un équilibre entre les conditions et les variations climatiques et l'expression des besoins ${ }^{*+4}{ }_{+}$

Un des premiers signes de la domestication sera le fait que la quotidienneté va se vivre en rapport avec les changements climatiques; elle se confinera de plus en plus à l'intérieur, à mesure que l'hiver approchera. Apparaît ensuite une forme systématisée de prévoyance: coupe du bois, fabrication d'outils, accumulation de réserves alimentaires (la boucherie), tissage et confection des vêtements. Pour contrer l'isolement, on organisera des "veillées"; la guignolée et les corvées seront également des signes de solidarité.

Les divertissements extérieurs sont aussi signes de maîtrise de l'hiver. Les glissades sur neige constituent la première activité, puis les Canadiens vont démontrer un engouement pour les courses en carrioles, qui avaient surtout lieu sur les ponts de glace, où tavernes et buvettes y étaient installées et servaient de lieu de rassemblement. Ces courses furent réglementees vers les milieu du 10 e siecle. Le patinage, une activité d'abord pratiquée par les ruraux sur le fleuve et les rivières gelées, fit une percée ver 1850 , au sein de l'élite citadine qui se retrouvera sur des patinoires ouvertes ou même fermées. Au $19 \mathrm{e}$ siecle, la traîne et le traineau, d'abord utilitaires, deviendront des jeux; il en va de même pour la raquette qui sera l'emblème des premiers clubs d'hiver et la pêche hivernale prendra l'aspect d'un véritable sport.

Cette évolution peut porter à penser que la réconciliation du Québécois avec l'hiver est accomplie et que les générations suivantes n'auront qu'à suivre la trace. Pourtant cet équilibre, encore très fragile, allait être rompu par l'urbanisation. La ville va attirer les populations, au point de parler d'exode, et vers 1900 , près de $50 \%$ de la population sera devenue urbaine: "le surpeuplement des quartiers ouvriers, l'exiguité des logements, le froid, la faim prolongent mềme dans des zones aux hivers plus cléments les angoisses de l'acclimatement et perpétuent la vulnérabilité de la domestication de $1^{\prime}$ hiver"s. Le mode de vie québecois est donc à repenser, toutes les activités quotidiennes, façonnées au rythme des saisons doivent être réorganisées, par exemple dans l'industrie il n"y a pas de retrait hivernal et tous les temps se suivent etse poursuivent. Que devientcette saison? Est-elle en train de perdre tout son sens? L'harmonie établie entre l'homme et la nature est-elle menacée? L'enracinement des habitants était fait, les signes naturels del'hiver persistaient; les Quebecois urbains partaient donc à la recherche d'un nouvel équilibre entre eux et cette froide saison.

\section{L'aménagement et les plaisirs de l'hiver}

Peut-on dire qu'aujourd'hui, il ne reste de l'hiver que les plaisirs? Bien sûr, quelques petits désagréments subsistent, comme de déblayage des rues et des autos, l'épandage de matières abrasives, l'ajout de survêtements. Pourtant, le Montréal sous-terrain et ses centaines de boutiques en particulier, ne réussissent-ils pas à faire passer sous silence l'hiver et son cortège d'intempéries? Lesdiverses techniques reliees au chauffage et à la conservation des aliments, par exemple, ne nous épargnent-elles pas des préparatifs de 1"hiver encore nécessaires il n"y a pas si longtemps? Certains y verront une maitrise parfaite de l'hiver, tandis que d'autres y sentiront davantage sa banalisation. Il m'apparaît évident que l'urbanisation, fille de la modernité, en nous faisant profiter de certains acquis, risque souvent de nous "dénaturés".

Enfin, de nouvelles pratiques sportives sont à l'honneur et elles attirent des touristes: en 1986-87, il y a eu 1013000 skieurs alpins, 1453000 skieurs de randonnée et 634000 motoneigistes dont la pratique a eté d'occasionnelle ( 1 à 4 jours durant la saison à régulière (15 jours ou plus durant la saison) ${ }^{6}$. De ce nombre, la clientèle venant de $1^{7}$ extérieur du Québec, se situe aux alentours de 20 a $22 \%$. La pratique des sports d'hiver est sans contredit l'une des spécificités de cette saison.

Mais une nouvelle rupture pointe $1^{+}$horizon de la culture hivernale québécoise, c'est ${ }^{44} 1$ 'effet de serre" provoquant le réchauffement de la planète. Nouveaudéfi! Comment s'adaptera-t-on à un hiver sans neige, par exemple qui éliminera peut-être les divertissements extérieurs actuels aux- quels nous sommes habitués?

\section{L'hiver québécois, pour combien de temps encore?}

Les spécialistes de l'environnement et les climatologues notent "qu'il est de mieux en mieux établi du point de vue scientifique que l'accroissement des "gas de serre" de 1'atmosphère pourrait entraîner, au cours des prochaines décennies, une augmentation de la température moyenne mondiale supérieure à toute autre augmentation survenue depuis l'arrivée de $1^{\text {thomme sur terre. }}$.

C'est pourquoi depuis 1978 , le Service de l'environnement atmosphérique d'Environnement Canada effectue de nombreuses études afin d'estimer les conséquences physiques, économiques et sociales de ces tendances sur notre environnement. Il est difficile, à l'heure actuelle, de prédire quand cela se fera sentir, mais dans le rapport Lamothe \& Périard, on y lit: "la comparaison des quantités moyennes de pluie et de neige entre la période 1975-85 et la période 1951 1980 montre que ces dix années ont eu en moyenne plus de pluie et moins de neige que les valeurs normales de trente ans" Cette même étude dira que: "Le nombre de jours skiables sur le sud du Québec diminuera de 50 à $70 \%$; pour les centres de ski équipés pour fabriquer de la neige artificielle, la réduction sera moins prononcée de 40 à $50 \%$. C'est ainsi qu'elle prévoit que dans les Laurentides, par exemple a Sainte-Agathe-des-Monts, "la période favorable au ski alpin ne débuterait qu'à Noẻl et se terminerait à la fin de février, soit un total de deux mois. $\mathrm{A}$ Sherbrooke, la période skiable irait du début janvier à la mi-février et finalement à Québec, la saison irait de la mi-décembre au début mars" 10 . Ces prévisions impliquent des stations équipées de canons à neige. Les auteurs conviennent que leur scénario est quelque peu sombre en raison des données et des études qui sont très nouvelles ${ }^{11}$. On peut donc penser que la tendance au réchauffement est commencée et va nous affecter, à plus ou moins long terme; alors les poudreries seront transformées en déluges, les patinoires rentreront toutes à l'intérieur, les "skidoos" deviendront des "seadoos" (d'ailleurs Bombardier a déjâ sa division de véhicules récréatifs d'eau), l'hiver gardera ses couleurs $\mathrm{d}^{\prime}$ automne avance, peintes de beige, de gris (du pâle au foncé) et de noir; fini le temps où le blane manteau de l'hiver nous enveloppait.

(à suivre à la page 25) 


\section{L'hiver québécois, un produit touristique?}

En premier lieu, rappelons que la notion de produit fait référence a l'industrie; or il ne fait aucun doute que le tourisme est une industrie récente, importante, mais quelque peu particulière, puisqu'elle fait commerce des patrimoines naturels et culturels. Fait assez étrange, on dit que l'industrie du tourisme comprend l'hếbergement, la restauration, le transport et parfois l'accueil; or toute cette infrastructure, bien qu'essentielle aux touristes, ne constitue absolument pas l'objet de leur déplacement. Lors de leurs voyages, les touristes veulent visiter une autre société et voir les "choses y devant être vues", ce sont là les attractions touristiques. Cen'est que depuis une quinzaine d'années qu'on commence à s'intéresserà cette composante de l'industrie touristique. Dean Mac Cannell dans The Tourist, fut sans doute le chef de file de ce nouveau courant de pensée. Il dira qu'un systeme d'attractions touristiques doit représenter la structure sociale d'une société, c'est-ầ-dire que chaque attraction touristique doit être représentative de l'une ou l'autre de ses caractéristiques.

Quant au produit touristique, disons brièvement, que c'est une ressource naturelle ou culturelle d'une société donnée, qu'on a démarquée, rehaussée, mise en scène, en un mot "sacralisée", pour en faire une "attraction" qui, contribuant al'identité culturelle de cette société, sera mis en marché pour attirer "l "étranger". En ce sens, une exposition Chagall, tenue à Montréal, même en hiver, est unévénement artistique important, mais pas une attraction touristique au sens propre du terme.

Le Québec hivernal $\mathrm{n}^{4}$ est pas vraiment une attraction en soi, c $^{+}$est beaucoup plus le mode de vie ou la façon dont les Québécois négocient avec cette saison qui l'est. Présentement, les pratiques sportives font l'attraction touristique hivemale, or il me semble que jusqu'à présent on a éprouvé certaines difficultés à sortir de ce créneau pour présenter l'hiver autrement. Lebulletin du Ministère du Tourisme "L'Actif" d'octobre 1989 a titré: "La publicité misera sur le ski et la motoneige". On précisera dans le texte, cependant que "Le Québec, c'est plus que le ski..." C'est pourquoi des renseignements ont été intégrés aux textes des annonces afind'attirer tous les amateurs de sports d'hiver ${ }^{12}$. Même en faisant un effort pour élargir la notion de "Tourisme hivernal $^{1+}$, on reste dans le même domaine.
Est-ce parce que l'hiver nous colle encore trop ă la peau, que nous avons du mal à imaginer d'autres façons de le rendre attirant? Il arrive parfois que dans le domaine du patrimoine, tant qu'une richesse est actuelle, on ne peut la mettre en exposition car elle fait trop partie de notre quotidien. L'hiverne serait-il pas l'occasion rêvée pour parler de nos campagnes et les présenter au monde urbanisé? Le tourisme d'aventure se prêterait bien à cette orientation.

Faudra-t-il attendre que l'effet de serre se soit accompli, tel que prévu et que la neige soit devenue un bien rare pour sacraliser notre mode de vie hivernal? L'hiver québecois ne deviendra-t-il une véritable attraction touristique patrimoniale que lorsqu”il aura disparu pour n'exister que dans notre mémoire collective.

On pensera alors à la "muséfifier" en créant un magnifique "Centre d'interpretation de l'hiver québécois". Sera-t-il alors plus agréable de vivre l'hiver à l'intérieur en se promenant de salle en salle où dans l'une. on présentera la tempête du siècle de mars 1972 , en vous soufflant un peu de neige artificielle; dans une autre, on vous fera essayer skis et patins; puis, on vous présentera des films sur la façon dont les Québécois ont apprivoisé ces temps? Nous. avons suffisamment de passé hivernal pour pouvoir le raconter. On pourrait ainsi attirer les touristes étrangers, même ceux du Sud, qui viendront voir, sentir et peut-être participer à nos hivers, comme à une aventure douce, forte, ou sportive, mais en tout cas spécifiquement québécoise. A l'aurore d'un point de rupture dans notre univers culturel, il faut repartir à la conquête d'une nouvelle hamonie dans les sillons mêmes de nos hivers passés.
Notes explicatives

(1) MACCANNELL, Dean, TheTourist, ANew theory of the Leisure Class, Schocken Books, New York, $1976,214 \mathrm{p}$.

(2) LAMONTAGNE, Sophie-Laurence, L'hiver dans la culture québécoise (XVI -XIX siécles). Institut quebecois de recherche sur la culture, Quebec $1983, p .25$.

(3) Idem, p. 32.

(4) Idem, p. 79.

(5) Idem, p. 161

(6) CLUZEAU, Patrick, Le tourisme hivernal des Québécois durant la saison d'hiver 19a6/1987. Les skieurs aloins, les motoneigistes, les skiews de randanné, Ministere du Tounisme, Direction de l'analyse et du developpement, Quebec 1988, pp. $41,82,118$.

(7) CEGIR, Leski alpin au Quebec: bilan et horizons. Québec, 1988, pp. $2,16$.

(8) Cf. Conférence de Villach. Australie, octobre 1985 dans Sommaire et changement climatique. Repercussions d'un changement cllmatiquesur IIndustrie du skialpin au Ouebec, Emironnement Canada, Avant-propos, 1988.

(9) Idem, p. 9 .

110) Idem, p. 5 .

(11) Idem, p. 1.

(12) L'ACTIF, Bulletin conjoint du Ministere du Tourisme et de l'ATRAa, wol, 1 , no $1,1989, p .1$. 